\title{
Espacialidad, tradición indígena y turismo sostenible: mirada sistémica de la cacería Sápara en Ecuador
}

\section{SPATIALITY, INDIGENOUS TRADITION AND SUSTAINABLE TOURISM: A SYSTEMIC LOOK AT THE SÁPARA HUNT IN ECUADOR}

\author{
SPATIALIDADE, TRADIÇÃO INDÍGENA E TURISMO SUSTENTÁVEL: \\ ASPECTO SISTÊMICO DA CAÇA SÁPARA NO EQUADOR
}

WLADIMIR Mejía Ayala ${ }^{1}$

Para citar este artículo: Mejía Ayala, W. (2017). Espacialidad, tradición indígena y turismo sostenible: mirada sistémica de la cacería Sápara en Ecuador. Perspectiva Geográfica, 22(2), 13-27. doi: 10.19053/01233769.7524
Recepción:

6 de marzo de 2017

Evaluación:

22 de agosto de 2017

Aprobación:

1 de septiembre de 2017

\section{Resumen}

Se pretende mostrar la recreación de la tradición indígena de la cacería Sápara como un sistema complejo y dinámico, el cual, al encontrarse inmerso en el espacio, permite responder a los retos que implica su turistificación sostenible y minimizar sus impactos negativos. La metodología empleada es de enfoque cualitativo, privilegiando la observación participante como método de recolección de la información. La experiencia in situ de esta tradición indígena de la Amazonía ecuatoriana se presenta en forma de relato. Como conclusión se propone que, desde el pensamiento sistémico, el turismo debe estar inmerso en la neguentropía, situación que se logra con una planificación desde las comunidades, basada en los límites del espacio físico en cuanto a su capacidad de reproducir en continuidad bienes y servicios, y en la capacidad de la comunidad local para resistir al señuelo del turismo y sus impactos.

1 Ingeniero agroforestal de la Universidad de Nariño, Máster en Espacio, Turismo y Sociedad de la Université Paris VII (Diderot), Máster en Gerencia de la cultura y desarrollo de prácticas turísticas sostenibles de la Université de Paris IX (Dauphine). Doctor en Geografía de la Université d'Angers, Francia. Coordinador académico (E). Estudios de Posgrado en Geografía (EPG). Programas Doctorado y Maestría en Geografía. Convenio Universidad Pedagógica y Tecnológica de Colombia e Instituto Geográfica Agustín Codazzi. wladimir.mejia@uptc.edu.co 
Palabras clave: espacialidad, teoría general de sistemas, tradición indígena, turistificación.

\section{Abstract}

The objective of this article is to show the recreation of the indigenous hunting tradition of the Sápara people as a complex and dynamic system that is inscribed in space, which responds to the challenges of sustainable tourism and minimizes negatives impacts. The methodology used is a qualitative approach, privileging participant observation as a method of data collection. The in situ traditional hunting experience of the Sápara people of the Ecuadorian Amazon is presented in the form of a story. In conclusion, it is proposed that, from a systemic perspective, tourism must be immersed in negentropy, which should be achieved through community planning. This should be based on the limits of the physical space in terms of its ability to reproduce goods and services, and the ability of the local community to resist the lure of tourism and its impacts.

Keywords: spatiality, general system theory, indigenous tradition, touristification.

\section{Resumo}

Pretendem-se amostrar a recreação da tradição indígena da caça Sápara como um sistema complexo e dinâmico, o qual, ao achar-se imerso no espaço, permite responder aos desafios que implica sua turistificação sustentável e minimizar seus impactos negativos. A metodologia utilizada é de enfoque qualitativo, privilegiando a observação participante como método de recolecção da informação. A experiência in situ desta tradição indígena da Amazônia equatoriana apresenta-se em forma de relato. Como conclusão se propõe que, desde o pensamento sistémico, o turismo deve estar imerso na neguentropia, situação que se alcança com uma planificação desde as comunidades, baseada nos limites do espaço físico em quanto a sua capacidade de reproduzir em continuidade bens e serviços e na capacidade da comunidade local para resistir à atração do turismo e seus impactos.

Palavras chave: espacialidade, teoria geral de sistemas, tradição indígena, turistificação. 


\section{Introducción.}

\section{La aproximación sistémica como prisma de observación}

A mediados del siglo XX, Ludwig von Bertalanffy (1968) señaló que el método científico, al estar basado en una visión mecanicista, causal y reduccionista, no permitía dar respuesta a ciertos problemas propuestos por los sistemas vivos. Esto lo llevó a plantear una reformulación global en el paradigma intelectual, lo que daría como resultado el surgimiento formal del pensamiento o mirada sistémica. La base filosófica del pensamiento sistémico es el holismo, es decir, la observación totalidades a partir de reglas aplicables a cualquier nivel de la realidad. Esto permite, tanto en el análisis de fenómenos como en las conclusiones resultantes, proponer soluciones en las cuales se tienen que considerar diversos elementos y relaciones que conforman la estructura de lo que se define como "sistema" y su entorno.

Según Von Bertalanffy (1968), un sistema es un complejo de elementos interactuantes, que, al estar abierto, interactúa al mismo tiempo con su entorno. Los elementos que conforman este complejo abierto pueden adquirir propiedades cualitativamente nuevas (emergentes), a través de su capacidad para auto-organizarse, por lo que están en una evolución continua. Esta evolución está relacionada con la entropía, que es la fuerza que todo sistema debe confrontar y que busca su desorganización. No obstante, gracias a un proceso de retroacción o de feed-back, un sistema es también capaz de producir neguentropía, o aquella energía que permite corregir la desviación producida por la entropía.

La mirada sistémica permite identificar con mayor claridad los fenómenos y sus posibles causas y consecuencias, facilitando su comprensión a partir de una reflexión inter, multi y transdisciplinaria. Desde esa perspectiva, y a partir del ejemplo de la cacería Sápara en la Amazonía ecuatoriana, este artículo propone la tesis de que al entender la recreación de una tradición como un sistema complejo y dinámico ${ }^{2}$ inmerso en una totalidad - representada por el espacio, entendido como medio ecológico (Santos, 1986) _ , se puede poner en valor el bien patrimonial inmaterial de un pueblo indígena, respondiendo a los retos que implica su turistificación y minimizando sus impactos negativos.

Es decir que, lejos de ser un simple espectáculo que atrae turistas, la recreación de una tradición indígena es un proceso ordenado de gestión sostenible y adaptativa de y en espacios altamente biodiversos, que está claramente reglamentado y el cual se desarrolla en continuidad en el tiempo. No obstante, en razón de la intangibilidad de la tradición, los objetos geográficos que participan en su recreación no están explícitos; el modo como esos objetos se muestran ante nuestros ojos es a partir de las relaciones que se establecen entre ellos y que pueden ser entendidos como procesos. Esta característica emergente puede, en un momento dado, favorecer la utilización responsable del patrimonio cultural inmaterial con fines turísticos y la creación de nuevos lugares para el desarrollo sostenible de esta actividad.

De esta manera, el artículo está dividido en cuatro partes. En la primera, se exponen las características socioespaciales del pueblo indígena Sápara y la

2 Según Morin (1990), la complejidad es un tejido de eventos, acciones, interacciones, retroacciones, determinaciones, que constituyen el mundo fenoménico y que desafían nuestras posibilidades de cálculo. Según el autor, la complejidad se presenta también con los rasgos inquietantes de lo enredado, de lo inextricable, del desorden, la ambigüedad, la incertidumbre y lo aleatorio. En palabras del autor, "la complejidad siempre está relacionada con el azar" (p. 60) 
manera como el turismo llega y se desarrolla en este territorio. Se presenta igualmente la metodología aplicada y se explica el por qué este pueblo fue tomado como caso de estudio. A continuación, en la segunda parte, se introduce el problema a partir de la narración de la experiencia in situ del autor y de su análisis, el cual se desarrolla, principalmente, apoyándose en la teoría general de sistemas de Bertalanffy (1968), y se presenta en la tercera parte. Finalmente, en la cuarta parte del artículo, se reflexiona sobre cómo a partir de la mirada sistémica, la recreación de una tradición indígena puede ser concebida de una manera más amplia, lo que puede traducirse en mejores oportunidades de las comunidades en el turismo.

\section{De la patrimonialización a la turistificación: el caso del pueblo Sápara del Ecuador}

El pueblo Sápara está conformado por una población de aproximadamente 1000 personas, de las cuales 200 tienen ascendencia étnica Sápara y solamente 5 hablan su lengua original. El resto de individuos tienen origen étnico Kichwa y Achuar o son mestizos. La población se encuentra dividida en 23 comunidades: las más pequeñas tienen una o dos familias, 14 personas aproximadamente; las medianas, un promedio de 8 familias, cerca de 50 personas; y la más grande, con 50 familias, alrededor de 300 personas. Las comunidades mantienen diferencias ideológicas que a veces pueden transformarse en manifestaciones violentas, que están relacionadas con la religión, el origen étnico, su relación con las petroleras y el poder sobre el territorio. No obstante, están reagrupadas, desde 2009, en una sola organización política, conocida como la Nacionalidad Sápara de Ecuador (NASE).
El pueblo indígena Sápara fue reconocido por la Unesco en 2001 como Obra Maestra del Patrimonio Cultural Inmaterial de la Humanidad y, en 2008, fue incluido en la lista representativa de este tipo de patrimonio. En 2009, de los dos pueblos indígenas de la selva amazónica que habían recibido un reconocimiento de la Unesco, el caso del pueblo Sápara era el único que registraba una actividad turística en su territorio ${ }^{3}$.

El territorio que habita el pueblo Sápara de Ecuador tiene una superficie de 370000 hectáreas. Se encuentra en la Alta Amazonía, en la parroquia Río Tigre, provincia de Pastaza, delimitado por los ríos Conambo y Pindoyaku. Estos ríos nacen al oeste de este territorio y no son navegables en la mayor parte de su recorrido. La reunión de sus cursos da nacimiento al río Tigre, en la frontera con el Perú, al extremo este del territorio. El área es accesible únicamente por vía aérea, después de 45 minutos de vuelo desde la parroquia de Shell; la ruta más cercana se encuentra a $120 \mathrm{~km}$ de distancia (Figura 1).

A pesar de este aislamiento, las incursiones de turistas en su territorio iniciaron a mediados de los años noventa y se intensificaron a inicios de los años dos mil, cuando el patrimonio oral y las manifestaciones culturales del pueblo Sápara fueron proclamados por la Unesco. Desde ese momento y sin considerar las dificultades para acceder, al menos dos pequeños grupos de entre tres y ocho personas, organizados

3 Solamente cuatro pueblos indígenas de la selva amazónica han logrado ser reconocidos por la Unesco como Patrimonio Cultural Inmaterial de la Humanidad: Ios pueblos Enawene Nawe de Brasil y Yurapari de Colombia en 2011. y los pueblos Wajapi de Brasil y Sápara de Ecuador y Perú, que en 2001 fueron primero declarados Obras-Maestras del patrimonio Oral e Inmaterial de la Humanidad y, en 2008, incluidos finalmente en la lista representativa. 
FIGURA 1.

Localización del territorio Sápara

Fuente: Mejía (2014)

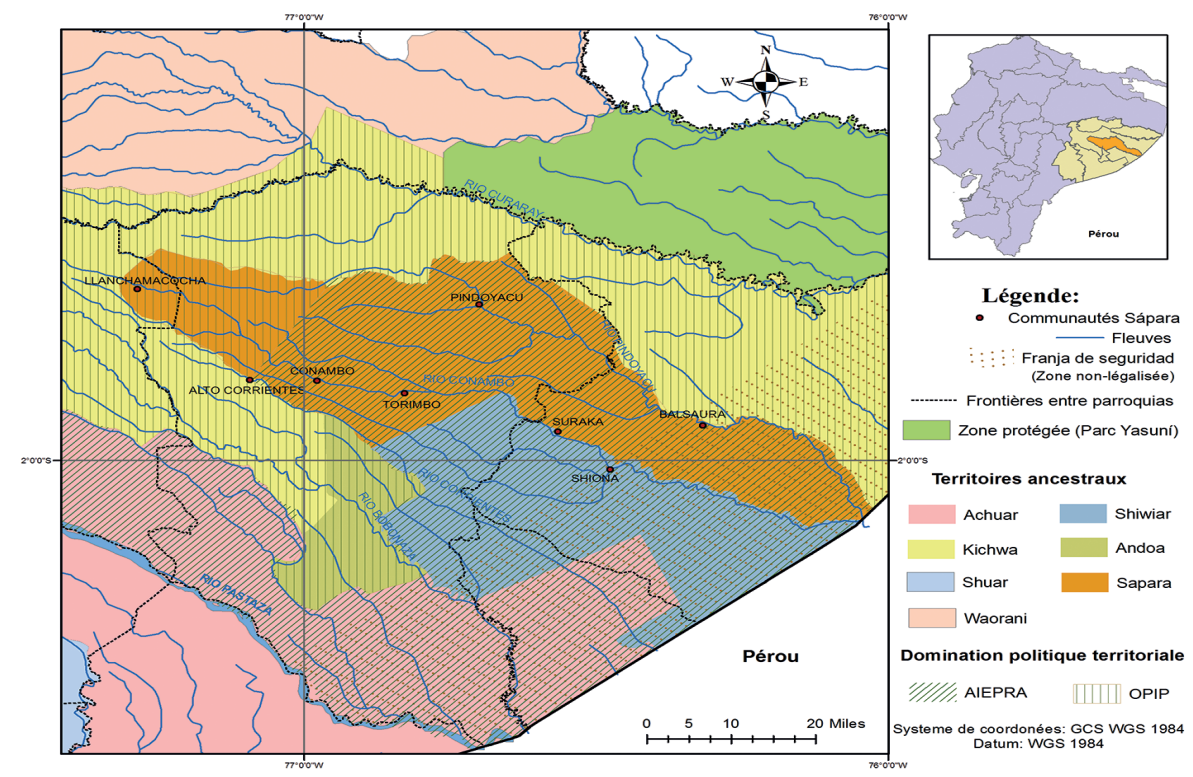

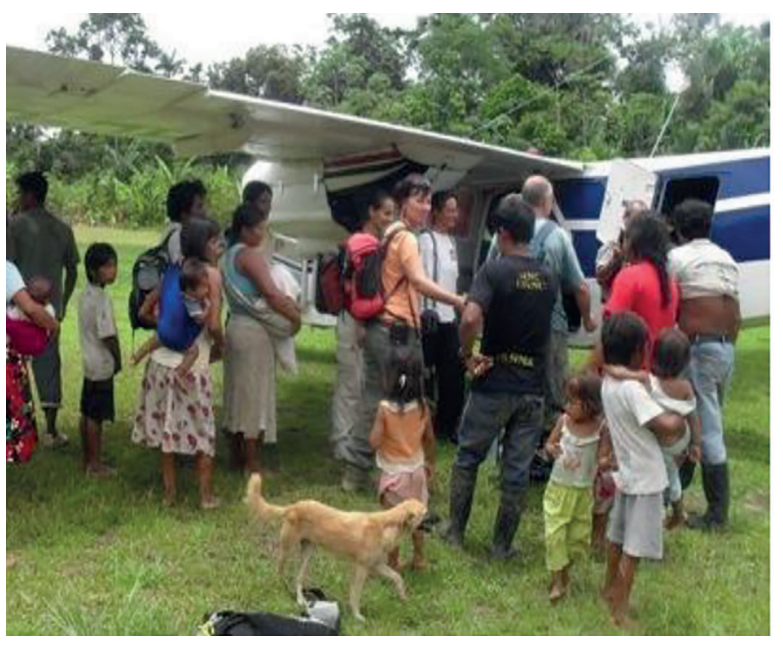

FIGURA 2.

Primer encuentro entre turistas y habitantes de la comunidad de Wiririma, territorio Sápara

Fuente: fotografía del autor, 18 de octubre de 2010

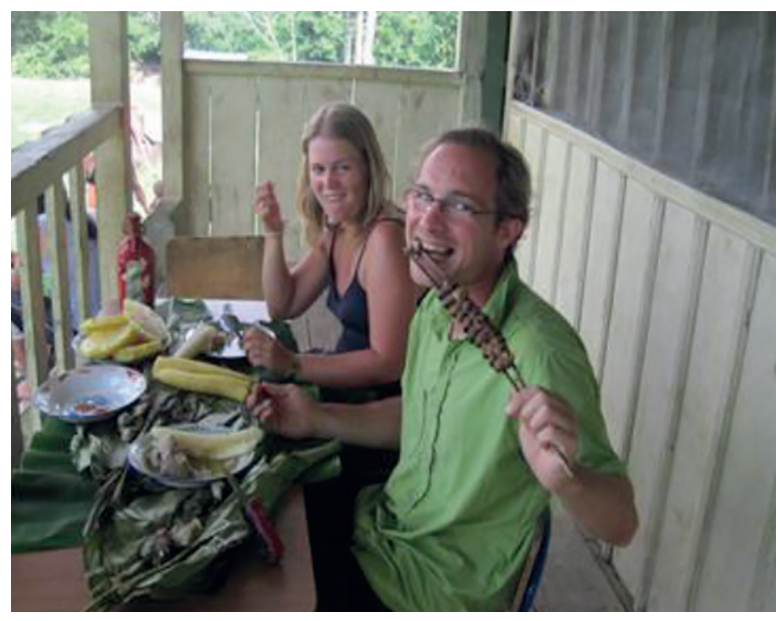

FIGURA 3.

Turistas descubren la gastronomía local con un plato compuesto por gusanos de palma asados y carne de caimán

Fuente: fotografía del autor, 22 de octubre de 2010.

No obstante, para comprender el origen y el desarrollo de este proceso, es necesario referirse a la industria petrolera. En Amazonía ecuatoriana, esta industria se desarrolló en los años setenta provocando significativos impactos socioambientales. Adiferencia de 
otros territorios indígenas, el territorio Sápara fue explorado por las compañías petroleras, pero no fue explotado. Buscando frenar una nueva irrupción, los Sápara fundaron nuevas comunidades sobre las fronteras de su territorio y cerca de los pozos identificados por dichas compañías. No obstante, la vida en las comunidades planteó nuevos retos a los Sápara, relacionados en gran parte con la llegada de la escolarización, que impuso en ellas un modo de vida sedentario.

Cuando las escuelas empezaron a funcionar, las familias que tenían niños en edad de escolarización debían permanecer en las comunidades al menos durante ocho meses al año. Esto significó que la producción agrícola, la cacería, la pesca y la recolección, realizadas anteriormente de manera itinerante a lo largo del territorio, se hicieran, desde ese momento, casi exclusivamente, en los alrededores cercanos de los caseríos. Esta situación fue observada por Bilhaut (2007), quien señaló que a medida que pasaba el tiempo, y en respuesta a la presión que se ejercía sobre este espacio, la variedad y la disponibilidad de alimentos decrecían, lo que hacía, paralelamente, cada vez más difícil la transmisión de conocimientos y creencias.

Paradójicamente, el hecho de tener una escuela en una comunidad, además de que esta estuviera equipada con una pista de aterrizaje y una radio de telecomunicaciones, o al menos estuviera localizada cerca de otra comunidad equipada con estos, creó las condiciones básicas que permitieron la llegada de turistas. Frente a una actividad petrolera contaminante y perturbadora a nivel de la sociedad ${ }^{4}$, y a una actividad agropecuaria imposible por las

4 En la década de los setenta, la industria petrolera se desarrolló en la parte norte de la Amazonía ecuatoriana. En este lugar, las compañías petroleras arrojaron los desechos resultantes de sus actividades en los ríos de los cuales los dificultades de llevar y comercializar la producción en los centros urbanos, el turismo fue concebido por los Sápara como la oportunidad de ganar, en algunos casos, por primera vez, un poco de dinero; pero también como un medio que permitía la conservación de su espacio de vida. Fue de esta manera que pequeños grupos de turistas comenzaron a recorrer su territorio.

En ausencia de un patrimonio cultural material, el territorio fue puesto en valor por los Sápara tanto como espacio natural, así como espacio cultural, a partir de la turistificación de sus tradiciones. Sin embargo, la entrada de tradiciones indígenas en el turismo y la evolución que esta actividad opera en ellas no es fácil de justificar, puesto que conlleva cuestionarse sobre su salvaguardia. La llegada de turistas a una comunidad que no contaba con servicios básicos, ni con gente formada en el turismo, ni con prácticas turísticas realmente establecidas, significó un gran impacto para los habitantes. Además de aceptar la presencia de extraños y su participación en la recreación de sus prácticas, se encontraban frente al riesgo de que esa intervención en su forma de vida transformara sus tradiciones

pueblos indígenas tomaban el agua. Hurtig y San Sebastián (2002) encontraron en comunidades indígenas pertenecientes a estos pueblos, aumento de casos de cáncer de estómago y de recto, en el caso de los hombres; de pelvis, en el de las mujeres; y de piel, en el caso de los niños. Esto se explica porque el petróleo en sí, contiene elementos tóxicos que, al no ser manipulados de manera correcta, producen cáncer: benceno, tolueno, metilbenceno y el xileno (BTEX); pero también porque la industria petrolera utiliza químicos que contienen metales pesados, como el cadmio y el bario, también considerados como peligrosos para la salud humana. Por otra parte, la construcción de carreteras y la llegada de colonos, provocó que miembros de pueblos indígenas se convirtieran en agricultores, ganaderos, comerciantes de carne y madera de la selva, o que se instalaran alrededor de los campos petroleros o a lo largo de las carreteras, dependiendo totalmente de las compañías extractivas. También provocó que miembros de los pueblos indígenas migaran a las ciudades en busca de mejores condiciones de vida. Todo esto favoreció la desarticulación de las familias y de las sociedades locales. 
FIGURA 4.

Localización de la comunidad de Suraka dentro del territorio Sápara

Fuente: Mejía (2014).

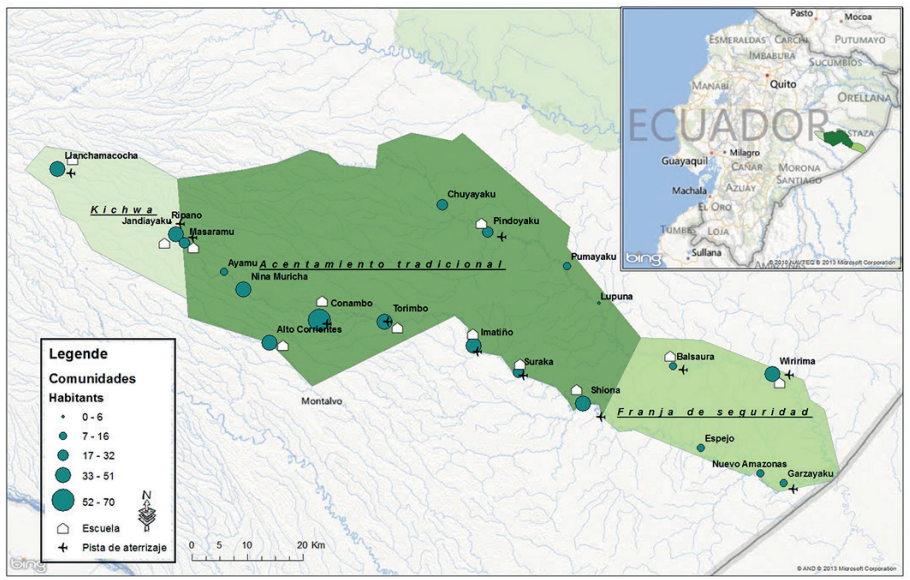

en objetos turísticos. Al trocar su valor de uso en valor de cambio, se obtenía como resultado un heritage commodification (Chambers, 2010), provocando consecuencias indeseables e irreparables en la comunidad.

Para ilustrar mejor esta situación, se presentará en el apartado siguiente y a partir del análisis de una experiencia personal in situ, el caso de la cacería Sápara y los problemas y retos que propone su turistificación. La metodología empleada —el prototurismo - es de enfoque cualitativo y la observación participante fue el método privilegiado para la recolección de la información. La experiencia in situ se presenta en forma de relato (extracto) y su análisis busca evidenciar la dificultad de turistificar la tradición indígena de la cacería.

\section{La cacería Sápara: construcción de un problema}

En octubre de 2010, durante el recorrido del territorio Sápara, llegué con un grupo de turistas a Suraka, una comunidad de 50 habitantes, localizada a orillas del río Conambo (Figura 4), donde se había previsto su participación en la recreación de la cacería. El objetivo era salir a la madrugada, recorrer un circuito y lograr abatir a un animal. Sin embargo, a la hora convenida, los cazadores no se presentaron.

Dos horas más tarde, cuando uno de los cazadores fue a dar una explicación sobre el incumplimiento de lo acordado con el grupo de turistas, simplemente nos dijo que había tenido "un mal sueño", añadiendo lo siguiente:

Para ir de cacería, hay que soñar primero andar al monte. Si en el sueño te dicen "sí", matas y traes los animales. Si estas mal soñado, no tienes que andar. A veces es peligroso de culebras. Así se va conociendo, soñando se aprende. Todas las noches se sueña de una u otra, de su pelada puede soñar. Los sueños de cacería es otra cosa, son muy diferentes de los otros. Primero hay que soñar para ir; sin soñar, sales y no te encuentras nada. Otra gente que viene aquí, también puede soñar, pero hay que decirle, tienes que soñar "así", para que conozcan nuestras costumbres. (Comunicación personal, 25 de octubre de 2010)

La anterior narración muestra, a partir del ejemplo de la cacería del pueblo Sápara, la dificultad para turistificar la recreación de una tradición indígena. Para comprender esta situación, se deben tener en cuenta dos características de la vida de los miembros de este pueblo indígena: su relación 
con los no humanos y la interpretación que ellos hacen de sus sueños. En cuanto a la primera, los Sápara consideran los no humanos, es decir, algunos animales y plantas, como personas (Bilhaut, 2007). Para ellos, los humanos y los no humanos disponen de un capital común de vida, de manera que todo exceso cometido en contra de una especie de fauna o de flora se traduce, necesaria e instintivamente, en una disminución de su propia esperanza de vida. Lévi-Strauss (1955), quien observó una situación similar en pueblos indígenas de la Amazonía brasileña, propuso que, desde la manera como los pueblos indígenas se concebían y entendían las relaciones con los no humanos, los hombres, los animales y las plantas podían ser considerados como elementos de un mismo sistema.

En cuanto a la segunda característica, muchos aspectos de la vida de los Sápara están basados en la interpretación de los sueños, y entre ellos se encuentra la cacería. Es así como todo cazador Sápara es susceptible de convertirse en el protector de una especie específica de animal, si, durante su sueño, encuentra, inicialmente con apariencia de humano, a este animal, quien, en sueños posteriores, se le presentará como el amo o maestro de la especie animal a la que pertenece, solicitándole ser su amigo. Según Bilhaut (2007), la relación onírica que se establece entre el soñador y el amo o maestro supone una solidaridad mutua tan fuerte que lleva al cazador no solamente a comprometerse a no cazar ni comer animales de esa especie, sino también a acogerlos y cuidarlos para siempre. A cambio, el amo o maestro onírico abastecerá al cazador de suficientes presas de cacería, comunicándole en un plano onírico el momento, el lugar, la especie y el número de animales a matar o, al contrario, advirtiéndole del peligro de entrar en la selva, logrando así que se aleje de su hogar. Es así como el sueño estructura la vida del cazador y, los resultados de la cacería, el hecho de matar animales para obtener el suficiente alimento para él y su familia, justifican las buenas relaciones mantenidas con su amigo onírico.

Estas características determinan el comportamiento de los Sápara; lo que permite explicar la experiencia no exitosa del grupo de turistas en la comunidad de Suraka. Es probable que, involuntariamente, la restricción que representa el mundo onírico de los Sápara para la turistificación de la tradición de la cacería impusiera una nueva situación beneficiosa para el turismo: "no es necesario matar los animales, solo deben observarse"; llevando a restar importancia a los sueños y a las relaciones mantenidas con los amigos oníricos, puesto que no tienen valor para el turismo, tratando más bien de dar un nuevo uso a los conocimientos y la experiencia de los cazadores dentro de un espacio que tiene las condiciones adecuadas para la conservación y observación de la fauna y la flora. De esta manera, matar animales a partir de la recreación de la cacería Sápara adquiere una connotación negativa.

Visto de manera superficial, el turismo se presenta como una actividad que favorece el desarrollo sostenible y permite salvaguardar el Patrimonio Cultural Inmaterial, puesto que genera ingresos económicos por primera vez para los locales, al mismo tiempo que los anima a conservar la fauna silvestre y pone en valor las técnicas y conocimientos acumulados y transmitidos durante milenios. No obstante, dejando de lado los sueños de cacería y las relaciones oníricas, el rol del turismo en este proceso propone una contradicción: esta actividad crea una distancia anteriormente inexistente entre humanos y no humanos. En efecto, el hecho de no matar animales no garantiza realmente evitar los 
excesos cometidos contra una especie determinada de animal, sino, paradójicamente, favorecerlos, lo que finalmente se traduce en una disminución de la esperanza de vida para los hombres mismos ${ }^{5}$. Así las cosas, la recreación de tradiciones indígenas como la cacería Sápara en ecosistemas frágiles como la Amazonía no es perjudicial, sino indispensable ${ }^{6}$.

\section{Espacialidad de la recreación de una tradición o cómo favorecer su utilización sostenible en el turismo}

Según Lussault (2003), si la conceptualización del espacio, a partir de sus características y atributos nos lleva a reflexionar teóricamente sobre las diferentes maneras como las sociedades lo organizan y se organizan en él, el concepto de espacialidad permite tomar en cuenta las acciones de quienes, a partir de diversos recursos y competencias espaciales, producen tales efectos. Más allá de la descripción del aspecto espacial de un fenómeno en el sentido de su repartición geográfica o como el simple resultado del movimiento de la sociedad, la espacialidad es pensada como la manera en que ese fenómeno se despliega en el espacio. Es decir, el fenómeno no se reduce a su sola localización

5 Algunas investigaciones desarrolladas por antropólogos y etnobiólogos como Berlín (1992), Balée (1993), Alvard (1993), incluso por Lévi-Strauss (1967), muestran el valor ecológico de las tradiciones indígenas tanto en el plano de la conservación como en el enriquecimiento de la biodiversidad amazónica.

6 Existen también detractores del rol de los pueblos indígenas en la conservación de espacios frágiles. Redford (1991), por ejemplo, nos previene sobre evitar caer de la trampa ideológica del "buen salvaje ecológico". Según el autor, los conocimientos y las tradiciones de los pueblos indígenas de la Amazonia, pueden estar obsoletas e, incluso, ser peligrosas. Sin embargo, el autor señala que una situación así puede presentarse cuando los pueblos indígenas están muy expuestos a la presión del mercado. al crecimiento demográfico y al sedentarismo. ni a su relación con otras realidades sociales, más que eso y tomando en cuenta su genealogía y sus múltiples formas ideales y materiales, la espacialidad es la manera como los objetos de la sociedad se asocian y ajustan, evidenciando una disposición particular adecuada a los actos del individuo o individuos concernidos.

De esta manera, cada individuo posee una espacialidad propia y específica, construida en la acción y que articula disposiciones espaciales variadas en una disposición global, la cual manifiesta al mismo tiempo su habitar ${ }^{7} \mathrm{y}$ constituye en tanto que configuración espacial estabilizada, interiorizada y vivida, su hábitat (Lussault, 2003). En el marco de este análisis, el espacio no es pensado como un contenedor neutro de funciones, ni como un bien comercial, ni como la superficie de proyección de las relaciones sociales, ni como un simple atributo de lo político, sino como una realidad construida en la acción espacial, que significa algo para alguien: para un actor ${ }^{8}$. Según Lussault (2003), al mismo tiempo que la autoriza, el espacio proviene de la espacialidad, y es por esto que se debe tratar desde el punto de vista de los actores que lo disponen: se desplazan en él, actúan e interactúan entre ellos y

7 El habitar, según Stock (2004), lejos de significar el simple hecho de residir, representa la manera por la cual una sociedad se desarrolla, se realiza o se despliega en actos. Es así como el espacio, en este caso el territorio del pueblo Sápara, es apropiado, donde, el término "apropiado" se lee en dos sentidos: primero, que pertenece a alguien; y luego, que ese alguien, a partir de su espacialidad, lo adaptó o apropió a su manera de habitar (Brunet, 1991).

8 Según Lussault (2003), un actor es un actante provisto de una interioridad subjetiva, de una intencionalidad, de una capacidad estratégica autónoma y de una capacidad enunciativa para manifestar su conformidad o desacuerdo. Esto implica, para el autor, que se encuentre inscrito en el espacio como un objeto identificable $y$, eventualmente, identificador, ya que, dentro de un funcionamiento colectivo, está cargado de valores comunes en los cuales pueden reconocerse, potencialmente pero no sistemáticamente, otros individuos. 
con otros actuantes, lo disputan, se afrontan, pero también disfrutan, viven y mueren.

Así las cosas, la espacialidad de la recreación de la tradición se transforma en un fenómeno complejo, al ser entendido como un proceso organizador que se da sobre un espacio ya constituido, para convertirlo en otro espacio de manera temporal. Acercarnos a la espacialidad de la recreación de la cacería Sápara, es entender que no se trata simplemente de abatir animales para satisfacer las necesidades alimentarias - como es frecuentemente concebido y juzgado por los turistas-. Esta tradición indígena representa uno de los grandes sistemas de gestión sostenible de la selva amazónica: es un sistema complejo, indivisible y dinámico, lo que le permite adaptarse a las condiciones siempre cambiantes del ambiente.

El hecho de pensar la recreación de esta tradición como un sistema permite concebirla como el proceso ordenado de puesta en relación de los elementos de ese sistema ${ }^{9}$ : desarrollándose en continuidad tanto en el espacio como en el tiempo, es decir, en diferentes lugares y momentos, y no solo durante el abatimiento del animal; es un proceso que permite finalmente

9 En la recreación de una tradición indígena, la puesta en relación de los elementos, responde a una reglamentación. Este conjunto de reglas, previamente establecido, es dinámico. A partir de su relación con el entorno, las reglas se actualizan y adaptan en continuidad, pero no se transforman. Estas reglas garantizan que se satisfagan las necesidades básicas de los habitantes de las comunidades, pero también otras necesidades más sutiles: mantener el equilibrio con el medio ambiente garantizando la cohesión de la sociedad local y su continuidad cultural como pueblo indígena, por ejemplo. La turistificación de una tradición indígena sugiere la necesidad u obligación de cuestionarse en cuanto a las reglas que deben imperativamente respetarse y aquellas que pueden ser objeto de una actualización o adaptación al entorno cambiante, o incluso, que pueden omitirse (Mejía, 2014). Es imperativo preguntarse ihasta qué punto se puede modificar su espacialidad, alejando su recreación de los lugares donde esta tenía lugar inicialmente, y cambiar sus actores iniciales (portadores) sin comprometer, a largo plazo, la conservación de sus territorios y su propia supervivencia física y cultural? considerar una participación más importante y sostenible del turismo en la recreación de esta tradición.

En efecto, como sistema, la cacería fue concebida o diseñada para cumplir una función: la de permitir la supervivencia física y cultural del pueblo Sápara. Para cumplir esta función, el sistema tiene necesidad de ciertos flujos de entrada, como los animales o presas de cacería, las plantas y la información; esta última representada por los conocimientos y saberes de los indígenas (Figura 5).

Los flujos de entrada son suministrados por el entorno o ambiente, con el cual, el sistema debe mantener buenas relaciones de reciprocidad ${ }^{10}$, y donde, según el principio de recursividad, la cacería como sistema se encuentra al mismo tiempo inmersa. Una vez al interior, los flujos de entrada son transformados en productos, servicios o información. Algunos de ellos serán retornados al entorno como flujos de salida. Cuando la calidad de esos flujos de salida corresponde a las expectativas y las capacidades del entorno, este los legaliza, es decir, considera que el sistema es viable y sostenible, por lo que le va a proveer una vez más materias primas (flujos de entrada), y así sucesivamente. Es por esta razón que no se puede considerar que el alimento obtenido del animal abatido represente el único flujo de salida del sistema, sino que se trata solamente de un producto, entre otros. En efecto, la manera por

10 Según Lévi-Strauss (1967), el principio de reciprocidad consiste en dar (o devolver) a aquel que nos ha dado. De esta manera, la reciprocidad figura, dentro de esta moralidad, como la obligación de ofrecer una compensación por los regalos o las ventajas recibidas. Según Malinowski (1922), el intercambio y la reciprocidad no están relacionados con intereses inmediatos, sino con la moralidad y la confianza que exige la vida en sociedad. Partiendo de esta idea, la operación de intercambio que se da a partir de la reciprocidad, puede ser entendida, en nuestro caso, como una permutación reversible de objetos (bienes) y de servicios, entre los sujetos inmersos en el sistema y el entorno. 
FIGURA 5.

La recreación de la tradición de la cacería como sistema

Fuente: Elaboración propia.

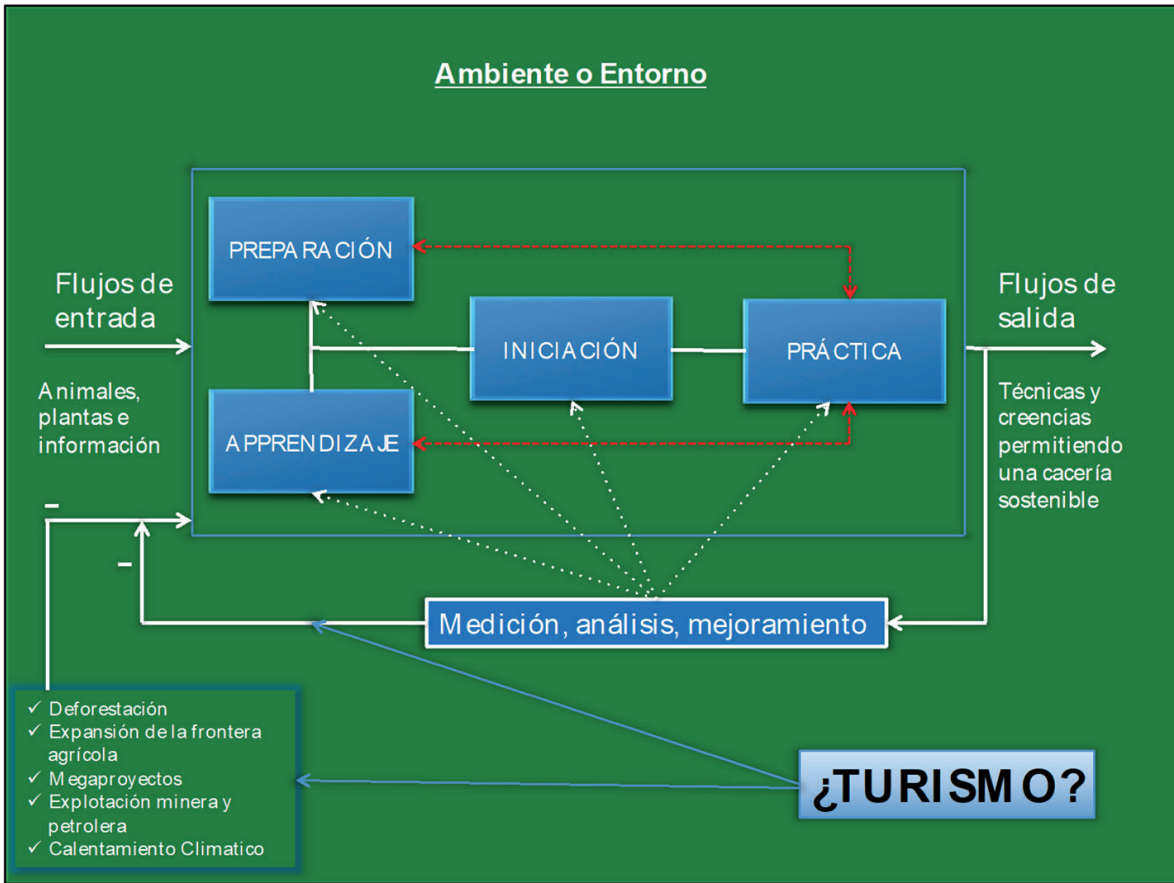

la cual se obtiene ese alimento es lo que representa verdaderamente el flujo de salida. Si esta manera de obtener el alimento es sostenible, el entorno suministrará nuevas materias primas al sistema.

Por otra parte, un sistema es definido como un conjunto de elementos o de procesos ligados los unos a los otros por relaciones de interdependencia. Al mismo tiempo, un proceso es definido como un encadenamiento ordenado de actividades permitiendo obtener un producto (Johansen, 2004). Es así como en la recreación de la tradición de la cacería Sápara se identifican cuatro procesos: la preparación del cazador, su aprendizaje, su iniciación y la práctica en sí misma. Dentro de una comunidad indígena, estos pueden desarrollarse el uno después del otro, o de manera simultánea, es decir, dos a la vez o, incluso, todos a la vez.

La preparación de un cazador (Figura 6) comienza en los primeros días de su nacimiento, con la ingestión de hojas de arbustos como el Brunfelsia chiricaspi y Maintenus krukori, las cuales, según los Sápara, tienen ciertas propiedades que permiten ver mejor en lo profundo de la selva, hacer desaparecer los miedos o adquirir la fuerza necesaria a nivel de la garganta para utilizar la cerbatana (Bilhaut, 2007). Con el fin de que las propiedades de estas plantas entren de la manera adecuada en el cuerpo del futuro cazador, el padre de este sopla el humo de tabaco (nicotina rustica) sobre el cuerpo de su hijo y su madre se somete a una dieta de carne durante al menos seis meses, tiempo durante el cual evita mantener relaciones sexuales. A la edad promedio de cuatro años, el niño ingiere de nuevo las hojas de estas plantas. En general, la preparación como proceso busca poner al futuro cazador en un estado en el cual él esté listo y disponible para aprender a cazar. Entre el conjunto de actividades que permiten este proceso se encuentran las de localizar las plantas en lo profundo de la selva y recolectar sus hojas e ingerirlas (existe la posibilidad de que estas plantas se produzcan en sus jardines). 
En cuanto a las principales actividades relacionadas con el proceso de aprendizaje, estas se desarrollan principalmente a la edad promedio de diez años, cuando el futuro cazador comienza a acompañar a su padre a los circuitos de cacería. Es con él que el niño aprende a caminar en la selva, evitando serpientes, identificando las presas adecuadas para ser abatidas, reconociendo todo tipo de plantas, animales e insectos que poseen diferentes virtudes, guardan significados espirituales o, incluso, sirven como referentes que alertan de la presencia de las presas o del camino que estas siguen.

Durante el aprendizaje el niño conoce igualmente las técnicas para fabricar armas, trampas y venenos de cacería, o, en el caso dado, aprende a obtenerlos de otros pueblos indígenas gracias a intercambios comerciales o tradicionales. Paralelamente, aprende a soñar, es decir, a interpretar los sueños de cacería. Es así como el aprendizaje, entendido como proceso, tiene como objetivo dotar al niño de las técnicas y conocimientos necesarios para cazar y para desarrollar una amistad sostenible con los maestros oníricos. El aprendizaje comporta así las actividades de formación concernientes a caminar en la selva, elaborar y maniobrar los objetos de cacería, interpretar los sueños y desarrollar relaciones con los maestros oníricos.

La iniciación, por su parte, es el ritual de paso de niño a adulto. Su objetivo como proceso es el de presentar a la sociedad un buen cazador, aquel que ha sido bien preparado, que ha aprendido de la mejor manera las técnicas de cacería y que puede mantener buenas relaciones con los maestros o amos de los animales, es decir, un individuo que está preparado para encontrar una mujer y crear una familia, garantizando así la sobrevivencia física y cultural del pueblo o grupo al cual pertenece. En cuanto a las actividades inmersas en este proceso, anteriormente cuando había chamanes, la iniciación incluía una toma de yagé (Banisteriopsis caapi); hoy, es el padre quien da de beber a su hijo jugo de tabaco y le impregna un poco en sus ojos (Bilhaut,

\section{FIGURA 6.}

Proceso de preparación

Fuente: Elaboración propia.

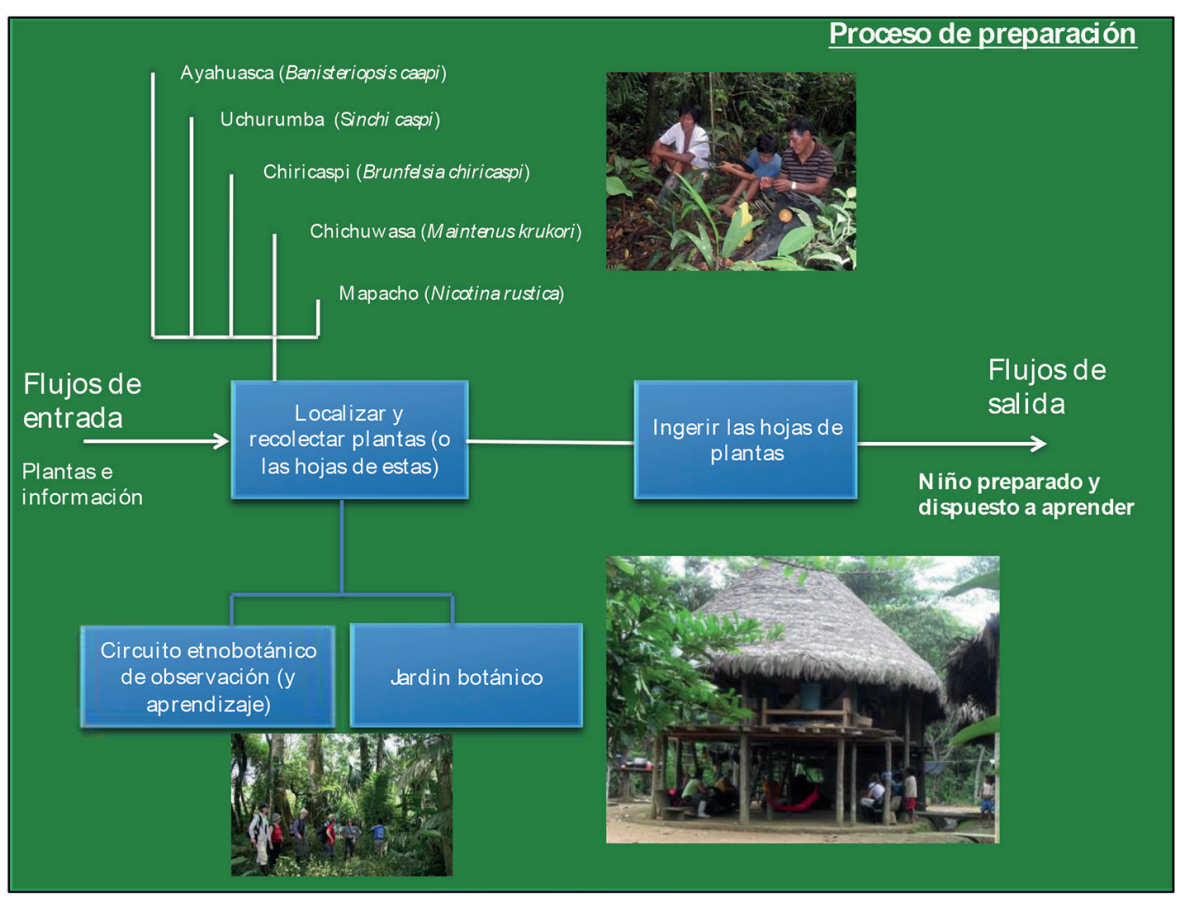


2007). Luego, el nuevo cazador se dirige, por primera vez y en solitario, a la selva profunda para cazar un animal. A su regreso, se organiza una fiesta con toda la comunidad para celebrar su victoria.

Finalmente, es en la práctica, entendida como proceso, donde, gracias a las buenas relaciones mantenidas con los maestros oníricos, se obtiene una presa. De esta manera, la práctica está conformada por las actividades de soñar, preparar los objetos de cacería, recorrer los circuitos y abatir al animal.

\section{Conclusión}

La mirada sistémica de la recreación de la tradición de la cacería Sápara muestra que cada actividad que se lleva a cabo en cada uno de los procesos se localiza en un lugar particular y, en la mayoría de los casos, en un momento diferente. Es en una gran parte de estas actividades y de lugares donde se encuentran las oportunidades de desarrollar el turismo de manera sostenible; puesto que son susceptibles de ser practicados por turistas sin intervenir, al menos, de manera violenta, en la tradición y en la forma de vida de las comunidades.

Sin embargo, es necesario recordar que todo sistema se enfrenta a una fuerza que busca su desorganización. Esta fuerza se llama entropía. En el caso de la recreación de la tradición de la cacería Sápara, la entropía corresponde a la presión del sistema económico capitalista, a la dependencia del dinero, al crecimiento descontrolado de la población y al sedentarismo, relacionados también con la deforestación, la expansión de la frontera agrícola, el desarrollo de megaproyectos, la explotación minera y petrolera y el calentamiento climático, o a todo aquello que implique el olvido (ver Figura 6). No obstante, gracias a un proceso de retroacción o de feed-back, un sistema es capaz de corregir la desviación producida por la entropía. La energía que permite hacerlo se llama neguentropía y es en esta donde el turismo deberá estar inmerso si se quiere lograr la utilización sostenible de una tradición.

Para eso, el desarrollo turístico debe ser planificado con las comunidades, basándose no solamente en los límites del espacio físico en cuanto a su capacidad de reproducir en continuidad bienes y servicios, sino también y sobre todo en la capacidad de la comunidad local para resistir al señuelo del turismo y sus impactos. Se trata de un turismo sostenible, planificado, que permita minimizar violaciones que podrían afectar a los cimientos mismos de su desarrollo, tales como la degradación de los ecosistemas y del patrimonio cultural o la ruptura de las tradiciones y estilos de vida, para dar entrada a servicios e infraestructura turística. El desarrollo del turismo sostenible en la Amazonía debe ser concebido como un desarrollo estático a nivel del espacio; la valoración consciente y responsable de los recursos del patrimonio cultural inmaterial se presenta como una herramienta importante para satisfacer las expectativas de los turistas.

Las comunidades deben formarse para acoger turistas, pero también deben sensibilizarse sobre los riesgos que el desarrollo de esta actividad implica frente a la importancia y fragilidad de sus tradiciones. De esta manera, las comunidades podrían escoger y establecer las actividades, los lugares y los periodos más pertinentes para permitir la participación de turistas. Es cierto que siempre existirá el riesgo de que la cotidianidad de las comunidades sea reinventada para satisfacer las necesidades de estos, no obstante, no se debe olvidar que la cacería no es más que una entre muchas otras tradiciones que están en constante recreación en las comunidades 
y territorios de pueblos indígenas. Esto permite de alguna manera pensar que es posible minimizar la probabilidad de perder las tradiciones en su conjunto, sin olvidar que sus aspectos culturales contemporáneos no son para nada menos atractivos que los tradicionales. Tal como lo proponen Lanfant,
Allcock y Bruner (1995), visto desde el interior de las comunidades, el turismo representa modernidad y cambio, pero, desde el exterior, es decir, desde la mirada del turista (Urry, 2002), la realidad de las comunidades indígenas es vista como exótica, primitiva e inmutable. 


\section{Referencias}

Alvard, M. (1993). Testing the "ecologically noble savage" hypothesis: Interspecific prey choice by Piro hunters of Amazonian Peru. Human Ecology, 21(4), 335-387. Recuperado de https://link.springer.com/article/10.1007/BF00891140

Balée, W (1993). Indigenous Transformations of Amazonien Forest: An exemple of Maranhão, Brazil. L'homme, 33(126), 231-254.

Berlin, B. (1992). Ethnobiological classification: principles of categorization of plants and animals in traditional societies. Princeton, N.J.: Princeton University Press. Recuperado de http://www.jstor.org/stable/j.ctt7ztq5q

Bilhaut, A. G. (2007). Le réveil de l'immatériel. La production du patrimoine onirique des Indiens Sápara (Haute Amazonie) (tesis de doctorado). Université Paris X Nanterre, Narrante, Francia. Recuperado de http://orbi.ulg.ac.be/handle/2268/139935

Brunet, R. (1991). Le territoire dans les turbulences. Montpellier: Geograpiques Reclus.

Chambers, E. (2010). Native Tours: The anthropoloy of travel and tourism. Long Grove, IL: Waveland.

Hurtig, A. K. y San Sebastián, M. (2002). Geographical differences in cancer incidence in the Amazon basin of Ecuador in relation to residence near oil fields. International Journal of Epidemiology, 31(5), 1021-1027. doi: 10.1093/ije/31.5.1021

Johansen, O. (2004). Introducción a la Teoría General de Sistemas. México: Limusa.

Lanfant, M. F., Allcock, J. y Bruner, E. (1995). Preface. En M. F. Lanfant, J. Allcock y E. Bruner. (Ed.), International Tourism. Identity and change. London: SAGE.

Lévi-Strauss, C. (1955). Tristes Tropiques. Paris: Plon.

Lévi-Strauss, C. (1967). Las estructuras elementales del parentesco. Barcelona: Paidós.

Lussault, M. (2003). Spatialité. En J. Lévy y M. Lussault, Dictionnaire de la géographie et de l'espace des sociétés (pp. 866-868). Paris: Belin.

Malinowski, B. (1922). Argonauts of the Western Pacific. An account of native enterprise and adventure in the Archipelagoes of Melanesian New Guinea. Londres: Routledge.

Mejía, V. (2014). Les problématiques et les enjeux de la mise en tourisme du patrimoine culturel immatériel: l'exemple du peuple indigène Sápara d'Équateur (tesis de doctorado). Universidad de Angers, Angers, Francia. Recuperado de http://www.theses.fr/s50152

Morin, E. (1990). Introduction à la pensée complexe. Paris: Seuil.

Redford, K. H. (1991). The ecologically noble savage. Cultural Survival Quarterly, 15,(1) 46-48. Recuperado de https://www.researchgate.net/publication/247848843 The_Ecologically_Noble_Savage

Santos, M. (1986). Espacio y Método. Geo Crítica, 12(65). Recuperado de http://www. ub.edu/geocrit/geo65.htm

Stock, M. (2004). L'habiter comme pratique des lieux géographiques. Recuperado de http:// www.espacestemps.net/articles/habiter-comme-pratique-des-lieux-geographiques/

Urry, J. (2002). The Tourist Gaze. London: SAGE.

Von Bertalanffy, L. (1968). General System theory: Foundations, Development, Applications. New York: George Braziller. 
